\title{
Accuracy of Two Stress Update Algorithms for Shear-Free Large Deformation Paths
}

\author{
By Antonio Rodríguez-Ferran ${ }^{1}$ and Antonio Huerta, ${ }^{2}$ Member, ASCE
}

\begin{abstract}
The behavior of two stress update algorithms for shear-free large deformation paths is analyzed. The first algorithm has a truncation error of order 1 . The second algorithm has a truncation error of order 2 . As a consequence, the global performance of the second algorithm is clearly superior. However, for the particular case of shear-free deformation paths, the first algorithm correctly predicts null shear stresses, while the second one does not. This behavior was reported in a previous paper for an extension-rotation test. In this note a general shear-free deformation path is considered in full detail.
\end{abstract}

\section{INTRODUCTION}

Stress update (i.e., time-integration of the constitutive equation) is a major concern in large strain solid mechanics. Several stress update algorithms can be found in the literature, see references cited by Rodríguez-Ferran et al. (1997) and Rodríguez-Ferran and Huerta (1998).

Two of these algorithms, originally presented by Bathe et al. (1975) and Pinsky et al. (1983), are considered in this work. Rodríguez-Ferran and Huerta (1998) showed that either of the two algorithms can be employed to upgrade a small strain finite-element code into large strains in a very simple manner. An accuracy analysis of the algorithms is presented by Rodríguez-Ferran et al. (1997). It is shown that the first algorithm (Bathe et al. 1975) has a truncation error (in time) of order 1, while the second algorithm (Pinsky et al. 1983) is of order 2 accuracy. As a consequence, the second algorithm typically produces clearly superior results, as shown by the numerical tests in the two papers by Rodríguez-Ferran et al. (1997) and Rodríguez-Ferran and Huerta (1998).

The order of accuracy of an algorithm is not the only relevant aspect. Depending on the application, other important issues must be considered. The behavior for shear-free deformation paths, a particular feature of the two algorithms, is analyzed in this paper.

\section{TWO STRESS UPDATE ALGORITHMS FOR LARGE STRAINS}

A detailed presentation of the two stress update algorithms can be found in the above-mentioned references. The final expressions are reviewed here.

\section{First Stress Update Algorithm}

The first stress update algorithm (Bathe et al. 1975) is

$$
{ }^{n+1} \boldsymbol{\sigma}={ }^{n} J^{-1}{ }^{n} \boldsymbol{\Lambda}{ }^{n} \boldsymbol{\sigma}{ }^{n} \boldsymbol{\Lambda}^{T}+{ }^{n} J^{-1}{ }^{n} \boldsymbol{\Lambda}\left(\mathbf{C}:{ }^{n} \Delta \mathbf{E}\right)^{n} \boldsymbol{\Lambda}^{T}
$$

where ${ }^{n} \boldsymbol{\sigma}$ and ${ }^{n+1} \boldsymbol{\sigma}=$ Cauchy stresses at times $t_{n}$ and $t_{n+1}=t_{n}$ $+\Delta t ;{ }^{n} \boldsymbol{\Lambda}=$ incremental deformation gradient relating configurations $\Omega_{n}$ and $\Omega_{n+1}$ (Fig. 1); ${ }^{n} J=\operatorname{det}\left({ }^{n} \boldsymbol{\Lambda}\right)=$ Jacobian of the

\footnotetext{
${ }^{1}$ Assoc. Prof., Departamento de Matemática Aplicada III, E.T.S. de Ingenieros de Caminos, Universitat Politècnica de Catalunya, Campus Nord C-2, E-08034, Barcelona, Spain.

${ }^{2}$ Prof., Departamento de Matemática Aplicada III, E.T.S. de Ingenieros de Caminos, Universitat Politècnica de Catalunya, Campus Nord C-2, E08034, Barcelona, Spain; corresponding author. E-mail: huerta@lacan. upc.es
}

incremental motion; $\mathbf{C}=$ elastic moduli tensor; and ${ }^{n} \Delta \mathbf{E}=$ incremental Lagrange strain tensor, defined as

$$
{ }^{n} \Delta \mathbf{E}=\frac{1}{2}\left({ }^{n} \boldsymbol{\Lambda}^{T}{ }^{n} \boldsymbol{\Lambda}-\mathbf{I}\right)
$$

In (1), the incremental deformation gradient ${ }^{n} \boldsymbol{\Lambda}$ is employed to push the stress forward at the beginning of the time-step, ${ }^{n} \boldsymbol{\sigma}$, and the stress increment, $\mathbf{C}:{ }^{n} \Delta \mathbf{E}$, from configuration $\Omega_{n}$ to configuration $\Omega_{n+1}$, where the stress at the end of the timestep, ${ }^{n+1} \boldsymbol{\sigma}$, is required.

This stress update algorithm is first-order accurate in time (i.e., the truncation error in the time-integration of the constitutive equation is $O(\Delta t)$ (Rodríguez-Ferran et al. 1997).

\section{Second Stress Update Algorithm}

The second stress update algorithm (Pinsky et al. 1983) is

$$
\begin{gathered}
{ }^{n+1} \boldsymbol{\sigma}={ }^{n} J^{-1}{ }^{n} \boldsymbol{\Lambda}{ }^{n} \boldsymbol{\sigma}{ }^{n} \boldsymbol{\Lambda}^{T}+{ }^{n+1 / 2} J^{-1}{ }^{n+1 / 2} \boldsymbol{\Lambda} \\
\left.\cdot\left(\mathbf{C}:{ }^{n+1 / 2} \Delta \boldsymbol{\varepsilon}\right)\right|_{n+1 / 2}{ }^{n+1 / 2} \boldsymbol{\Lambda}^{T}
\end{gathered}
$$

where $^{n+1 / 2} \boldsymbol{\Lambda}=$ incremental deformation gradient relating the midstep configuration $\Omega_{n+1 / 2}$ to the end-of-step configuration $\Omega_{n+1}$ (Fig. 1); the Jacobian ${ }^{n+1 / 2} J$ is defined as $\operatorname{det}\left(\Omega_{n+1 / 2}\right)$; and the midstep strain increment ${ }^{n+1 / 2} \Delta \boldsymbol{\varepsilon}$ is defined as

$$
{ }^{n+1 / 2} \Delta \boldsymbol{\varepsilon}=\frac{1}{2}\left\{\left[\frac{\partial(\Delta \mathbf{u})}{\partial\left(^{n+1 / 2} \mathbf{x}\right)}\right]+\left[\frac{\partial(\Delta \mathbf{u})}{\partial{\left({ }^{n+1 / 2} \mathbf{x}\right)}^{T}}\right]^{T}\right\}
$$

with $\Delta \mathbf{u}$ the displacement increment; and ${ }^{n+1 / 2} \mathbf{x}$ the midstep coordinates.

This stress update algorithm is second-order accurate in time [i.e., the truncation error in the time-integration of the constitutive equation is $O\left(\Delta t^{2}\right)$ (Rodríguez-Ferran et al. 1997].

\section{ACCURACY FOR SHEAR-FREE DEFORMATION PATHS}

A particular behavior of the first stress update algorithm is demonstrated here. This algorithm correctly predicts null shear stresses for any shear-free deformation path. The second algorithm, on the contrary, only provides similar results for some particular deformation paths of this type.

The general expression for shear-free deformation paths is

$$
\begin{aligned}
& x(t)=X a_{x}(t) \cos \theta(t)-Y a_{y}(t) \sin \theta(t) \\
& y(t)=X a_{x}(t) \sin \theta(t)+Y a_{y}(t) \cos \theta(t)
\end{aligned}
$$

where $a_{x}(t)$ and $a_{y}(t)$ represent the axial deformations in the $x$ and $y$ directions, respectively; and $\theta(t)=2 \pi t$ accounts for the rigid rotation. Taking $a_{x}(t)=1+t$ and $a_{y}(t)=1$ yields an extension-rotation test. An extension-compression-rotation test 


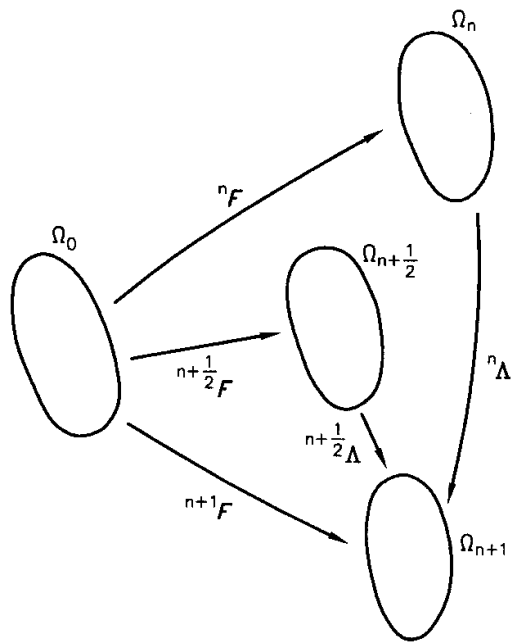

FIG. 1. Deformation Gradients in Incremental Analysis

with no change in volume can be obtained with $a_{x}(t)=1 / a_{y}(t)$ $=1+t$, and choosing $a_{x}(t)=a_{y}(t)=1+t$ renders a dilatationrotation test. To assess the behavior of the two stress update algorithms, it is enough to study the stress predicted after one time-step, and to check if the shear stress component (in rotated axes, to account for the rigid rotation) is null or not.

\section{First Stress Update Algorithm}

For the first time-step $\Delta t$, the incremental Lagrange strain tensor is $[(2)]$

$$
\Delta \mathbf{E}=\frac{1}{2}\left[\begin{array}{cc}
\left(A_{x}^{2}-1\right) & 0 \\
0 & \left(A_{y}^{2}-1\right)
\end{array}\right]
$$

with $A_{x}=a_{x}(\Delta t)$; and $A_{y}=a_{y}(\Delta t)$. Recalling the expression of the first algorithm (1), the stress after one time-step is

$$
\begin{aligned}
\boldsymbol{\sigma} & =\frac{E}{2 A_{x} A_{y}} \\
& \cdot\left[\begin{array}{cc}
\left(B_{x} \cos ^{2} \Theta+B_{y} \sin ^{2} \Theta\right) & \left(B_{x} \sin \Theta \cos \Theta-B_{y} \sin \Theta \cos \Theta\right) \\
\left(B_{x} \sin \Theta \cos \Theta-B_{y} \sin \Theta \cos \Theta\right) & \left(B_{x} \sin ^{2} \Theta+B_{y} \cos ^{2} \Theta\right)
\end{array}\right]
\end{aligned}
$$

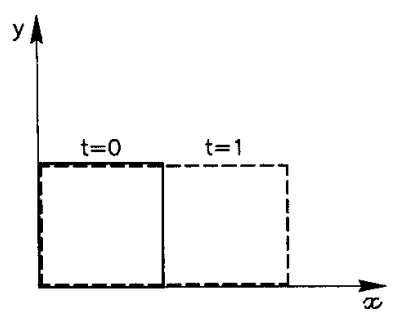

(a)

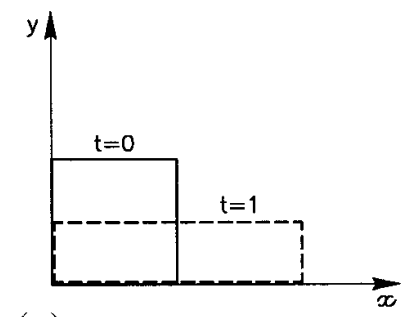

(c) with $\Theta=2 \pi \Delta t ; B_{x}=A_{x}^{2}\left(A_{x}^{2}-1\right)$; and $B_{y}=A_{y}^{2}\left(A_{y}^{2}-1\right)$.

If the stress $\boldsymbol{\sigma}$ is transformed into the rotated stress $\boldsymbol{\sigma}^{\prime}=$ $\mathbf{R} \boldsymbol{\sigma} \mathbf{R}^{T}$, where $\mathbf{R}$ is an orthogonal tensor that accounts for the rigid rotation, the result is

$$
\boldsymbol{\sigma}^{\prime}=\frac{E}{2}\left[\begin{array}{cc}
\frac{A_{x}}{A_{y}}\left(A_{x}^{2}-1\right) & 0 \\
0 & \frac{A_{y}}{A_{x}}\left(A_{y}^{2}-1\right)
\end{array}\right]
$$

Eq. (6) shows that null shear stresses $\sigma_{x^{\prime} y^{\prime}}$ are predicted, independently of the axial deformations $A_{x}$ and $A_{y}$. It can easily be checked that the analysis just performed for the first timestep can be extended to the successive increments. In conclusion, the first algorithm predicts null shear stresses (in the rotated axes) $\sigma_{x^{\prime} y^{\prime}}$ for any number of time-steps and for any choice of $a_{x}(t)$ and $a_{y}(t)$.

\section{Second Stress Update Algorithm}

A similar analysis has been performed for the second algorithm. Since the midstep configuration is employed, the required computations are rather cumbersome in this case. The incremental displacements for the first time-step can be computed from (5). After that, the midstep coordinates ${ }^{n+1 / 2} \mathbf{X}$ are obtained via linear interpolation. Then the incremental displacements $\Delta \mathbf{u}$ are written in terms of the midstep coordinates, so the strain increment ${ }^{n+1 / 2} \Delta \boldsymbol{\varepsilon}$ [(4)], can be written as

$$
\begin{aligned}
& { }^{n+1 / 2} \Delta \boldsymbol{\varepsilon}=\frac{2}{A_{x} A_{y}+\left(A_{x}+A_{y}\right) \cos \Theta+1} \\
& \quad \cdot\left[\begin{array}{cc}
A_{x} A_{y}+\left(A_{x}-A_{y}\right) \cos \Theta-1 & \left(A_{x}-A_{y}\right) \sin \Theta \\
\left(A_{x}-A_{y}\right) \sin \Theta & A_{x} A_{y}-\left(A_{x}-A_{y}\right) \cos \Theta-1
\end{array}\right]
\end{aligned}
$$

Once the strain increment is known, the stress $\boldsymbol{\sigma}$ is computed by employing the incremental deformation gradients (3), and then transformed into the rotated stress $\boldsymbol{\sigma}^{\prime}$. The final result is that the shear stress $\sigma_{x^{\prime} y^{\prime \prime}}$ is proportional to a factor that depends on axial deformations $A_{x}$ and $A_{y}$

$$
\sigma_{x^{\prime} y^{\prime}} \sim-A_{x}^{3} A_{y}^{2}+A_{x}^{2} A_{y}^{3}+A_{x}^{2} A_{y}-A_{x} A_{y}^{2}
$$

It can be seen from (7) that $\sigma_{x^{\prime} y^{\prime}}$ is not null for any choice of $a_{x}(t)$ and $a_{y}(t)$. Taking $a_{x}(t)=1+t$ and $a_{y}(t)=1$ (extension-

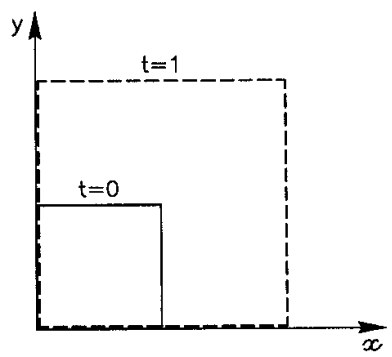

(b)

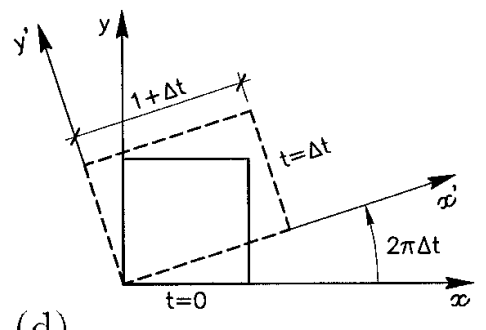

(d)

FIG. 2. Shear-Free Large Deformation Paths: (a) Uniaxial Extension; (b) Dilatation; (c) Extension and Compression; (d) Extension and Rotation 
TABLE 1. Observed Order of Convergence for Various Tests

\begin{tabular}{l|c|c|c|c|c|c}
\hline \hline \multirow{2}{*}{\begin{tabular}{c}
\multirow{2}{*}{ Test } \\
$(1)$
\end{tabular}} & \multicolumn{3}{|c|}{ OBSERVED ORDER OF CONVERGENCE } \\
\cline { 2 - 7 } & \multicolumn{2}{|c|}{$\boldsymbol{\sigma}_{x x}$} & \multicolumn{2}{c|}{$\boldsymbol{\sigma}_{x y}$} & \multicolumn{2}{c}{$\boldsymbol{\sigma}_{y y}$} \\
\cline { 2 - 7 } & $\nabla$ & $\triangle$ & $\nabla$ & $\triangle$ & $\nabla$ & $\triangle$ \\
& $(2)$ & $(3)$ & $(4)$ & $(5)$ & $(6)$ & $(7)$ \\
\hline Uniaxial extension & 1.13 & 1.95 & Exact & Exact & Exact & Exact \\
Dilatation & 1.11 & 1.97 & Exact & Exact & 1.11 & 1.97 \\
Extension and compression & 1.15 & 1.93 & Exact & Exact & 0.87 & 1.99 \\
Extension and rotation & 1.07 & 2.30 & Exact & 2.57 & Exact & 4.72 \\
\hline Note: $\nabla=$ first algorithm; $\triangle=$ second algorithm. \\
\hline \hline
\end{tabular}

rotation test), for instance, yields a non-null $\sigma_{x^{\prime} y^{\prime}}$. On the contrary, for $a_{x}(t)=a_{y}(t)$ (dilatation-rotation test) and for $a_{x}(t) a_{y}(t)$ $=1$ (extension-compression-rotation test, with no volume change), null values for $\sigma_{x^{\prime} y^{\prime}}$ are predicted. In conclusion, the second stress update algorithm predicts correct null shear stresses (in the rotated axes) for some particular deformation paths of the form given by (5), but not for every shear-free path.

\section{NUMERICAL EXAMPLES}

The two stress update algorithms have been tested for various shear-free large deformation paths (Rodríguez-Ferran et al. 1997; Rodríguez-Ferran and Huerta 1998) [Figs. 2(a-d)].

Both elastic and plastic computations have been performed, with different values of the time-step $\Delta t$. The output of the elastic computations is summarized in Table 1. For each test and each stress component, the observed order of convergence is computed by comparing the analytical solutions with the numerical solutions. The first algorithm correctly predicts null values of the shear stress. The second algorithm, on the other hand, yields spurious shear stresses for the extension and rotation test.

\section{CONCLUDING REMARKS}

The performance of two stress update algorithms for large deformation paths without shear strains has been analyzed. The analysis and the numerical experiments show that the algorithm with a truncation error of order 1 correctly predicts null shear stresses for any shear-free deformation path. The second-order algorithm, on the other hand, yields spurious shear stresses for some shear-free deformation paths. This result clearly shows that the global time accuracy is not the only concern when selecting a stress update algorithm, and that other issues must be considered for specific applications. If the basic goal is the global accuracy of the stress tensor (i.e., considering all the components), then the second-order algorithm is preferable, because the error in the shear stresses is more than compensated for by the higher accuracy in the normal stresses. However, if shear stresses are a key factor in the analysis, then the first-order algorithm is better.

\section{ACKNOWLEDGMENTS}

The financial support of the Ministerio de Educación y Cultura of Spain (Grant No. TAP98-0421) is gratefully acknowledged.

\section{APPENDIX. REFERENCES}

Bathe, K. J., Ramm, E., and Wilson, E. L. (1975). "Finite element formulations for large deformation dynamic analysis." Int. J. Numer. Methods in Engrg., 9, 353-386.

Pinsky, P. M., Ortiz, M., and Pister, K. S. (1983). "Numerical integration of rate constitutive equations in finite deformation analysis." Comp. Methods Appl. Mech. Engrg., 40, 137-158.

Rodríguez-Ferran, A., Pegon, P., and Huerta, A. (1997). "Two stress update algorithms for large strains: Accuracy analysis and numerical implementation.' Int. J. Numer. Methods in Engrg., 40, 4363-4404.

Rodríguez-Ferran, A., and Huerta, A. (1998). "Comparing two algorithms to add large strains to a small strain finite element code.' J. Engrg. Mech., ASCE, 124, 939-948. 